\title{
ANÁLISE DAS MEDIDAS AERODINÂMICAS NO PORTUGUÊS BRASILEIRO POR MEIO DO MÉTODO MULTIPARAMÉTRICO DE AVALIAÇÃO VOCAL OBJETIVA ASSISTIDA (EVA)
}

\section{Analysis of aerodynamic measures in Brazilian portuguese through Multiparameter Assessment Method Vocal Objective Assist (EVA)}

\author{
Karina Vitor de Oliveira ${ }^{(1)}$, Bárbara Silveira de Faria ${ }^{(2)}$, Juliana Preisser Godoy e Silva ${ }^{(3)}$, \\ César Reis ${ }^{(4)}$, Alain Ghio ${ }^{(5)}$, Ana Cristina Côrtes de Gama ${ }^{(6)}$
}

\begin{abstract}
RESUMO
Objetivo: definir medidas aerodinâmicas em falantes do português brasileiro, sem queixas vocais, obtidas pelo programa EVA. Método: participaram do estudo 20 homens e 20 mulheres, que tiveram suas vozes analisadas. Resultados: os valores médios referentes à pressão subglótica para vozes femininas e masculinas foram, respectivamente: Pressão subglótica= 5,84 hPa e 6,7 hPa, média da intensidade $=79,21 \mathrm{~dB}$ e $81,7 \mathrm{~dB}$, média do fluxo oral=0,09 dm3 $/ \mathrm{s}$ e $0,16 \mathrm{dm}^{3} / \mathrm{s}$, eficiência glótica $=$ $13,87 \mathrm{~dB} / \mathrm{hPa}$ e $12,78 \mathrm{~dB} / \mathrm{hPa}$, eficiência laríngea $=188,08 \mathrm{~dB} /\left(\mathrm{hPa} \cdot \mathrm{dm}^{3} / \mathrm{s}\right)$ e $97,11 \mathrm{~dB} /\left(\mathrm{hPa} \cdot \mathrm{dm}^{3} / \mathrm{s}\right)$, resistência laríngea $=96,26 \mathrm{hPa} /\left(\mathrm{dm}^{3} / \mathrm{s}\right)$ e $52,64 \mathrm{hPa} /\left(\mathrm{dm}^{3} / \mathrm{s}\right)$, média da frequência fundamental $(\mathrm{FO})=$ $208,28 \mathrm{~Hz}$ e $136,56 \mathrm{~Hz}$ e pico da pressão do fluxo oral=0,093 e 0,098. Houve diferença estatisticamente significante em relação ao gênero para as medidas de média de intensidade, eficiência laríngea, resistência laríngea e média da frequência fundamental. Os valores médios referentes ao fluxo oral para vozes femininas e masculinas foram respectivamente: Pressão subglótica= 6,05 hPa e 6,6 $\mathrm{hPa}$, média da intensidade $=65,50 \mathrm{~dB}$ e $66,3 \mathrm{~dB}$, média do fluxo oral $=0,10 \mathrm{dm}^{3} / \mathrm{s}$ e $0,13 \mathrm{dm}^{3} / \mathrm{s}$, eficiência glótica $=11,12 \mathrm{~dB} / \mathrm{hPa}$ e $11,77 \mathrm{~dB} / \mathrm{hPa}$, eficiência laríngea $=144,83 \mathrm{~dB} /\left(\mathrm{hPa} \cdot \mathrm{dm}^{3} / \mathrm{s}\right)$ e 97,89 dB/ $\left(\mathrm{hPa} \cdot \mathrm{dm}^{3} / \mathrm{s}\right)$, resistência laríngea $=78,98 \mathrm{hPa} /\left(\mathrm{dm}^{3} / \mathrm{s}\right)$ e $61,81 \mathrm{hPa} /\left(\mathrm{dm}^{3} / \mathrm{s}\right)$, média da $\mathrm{F} 0=222,52 \mathrm{~Hz}$ e $139,20 \mathrm{~Hz}$ e pico da pressão do fluxo oral= 0,047 e 0,053. Conclusão: o EVA é um programa ainda novo no Brasil, e a análise de medidas aerodinâmicas, em falantes do português brasileiro, permite a obtenção de valores de referência, possibilitando assim comparações com estudos futuros.
\end{abstract}

DESCRITORES: Voz; Pressão do Ar; Acústica da Fala; Fonoaudiologia

(1) Acadêmica de Fonoaudiologia da Universidade Federal de Minas Gerais - UFMG - Belo Horizonte (MG), Brasil.

(2) Acadêmica de Fonoaudiologia da Universidade Federal de Minas Gerais - UFMG - Belo Horizonte (MG), Brasil.

(3) Fonoaudióloga; Mestre em Linguística pela Universidade Federal de Minas Gerais - UFMG.

(4) Linguista; Professor Associado da Faculdade de Letras da Universidade Federal de Minas Gerais - UFMG - Belo Horizonte, MG, Brasil.

(5) Engenheiro; Pesquisador do Laboratório de Fala e Linguagem da Universidade Aix-Marseille, Aix-en-Provence, França.

(6) Fonoaudióloga; Professora Associada do Departamento de Fonoaudiologia da Universidade Federal de Minas Gerais UFMG, Belo Horizonte, MG, Brasil.

Conflito de interesses: inexistente

\section{INTRODUÇÃO}

A análise acústica é uma forma de avaliação da voz que vem contribuindo para a determinação de parâmetros de normalidade, por ser uma avaliação objetiva e não invasiva, que possibilita o armazenamento de dados para posteriores análises e comparações. O desenvolvimento de programas específicos possibilitou o aumento da precisão do diagnóstico e a identificação dos resultados do tratamento a curto e longo prazo ${ }^{1}$. Apesar do inquestionável avanço que a análise acústica trouxe ao diagnóstico e tratamento dos problemas de voz, é importante ressaltar que medidas acústicas são complementares e não substitutas das avaliações clínicas subjetivas². 
Entre os parâmetros avaliados na análise acústica estão as medidas de aerodinâmica da fala, como a pressão subglótica e o fluxo oral, que são valiosas medidas utilizadas na investigação e avaliação clínica da função laríngea² ${ }^{2}$. Os pulmões servem como uma fonte de pressão constante durante a fonação, e essa pressão pode ser utilizada para determinar a eficiência com que a laringe converte a energia aerodinâmica para energia acústica ${ }^{3,4}$. À medida que a laringe funciona como um transdutor de aerodinâmica, a eficiência vocal, definido como potência sonora $(\mathrm{dB})$, dividido pela energia aerodinâmica (fluxo de oral), indica as condições da saúde vocal ${ }^{4}$.

A avaliação exata dos parâmetros aerodinâmicos, facilmente utilizados na prática clínica, pode permitir de forma não invasiva a detecção da patologia do trato vocal e fornecer "feedback" sobre a eficácia do tratamento após diagnóstico ${ }^{5}$. Medições de resistência glótica ou resistência laríngea podem fornecer informações sobre as características físicas das vias aéreas, bem como as propriedades mecânicas dos tecidos da laringe ${ }^{6}$. A resistência é dependente do tamanho da via aérea, e, vários outros fatores contribuem para resistência glótica, incluindo o grau de adução das pregas vocais e a velocidade das partículas de ar através da glote ${ }^{6}$.

Pelo fato de os processos aerodinâmicos serem responsáveis por parte dos mecanismos de produção da fala, tal método constitui uma importante ferramenta no diagnóstico e acompanhamento do tratamento cirúrgico de pacientes com insuficiência velofaríngea. No que se refere ao mecanismo velofaríngeo, a técnica permite inferir o potencial anatômico da velofaringe durante a fala ${ }^{7}$.

O Laboratoire Parole et Langage da Universidade de Aix-en-Provence - França - desenvolveu o Método Multiparamétrico de Avaliação Vocal Objetiva Assistida (EVA), o qual utiliza o processador de dados SESANE, destinado a analisar a fala normal e/ou com alteração. O sistema de avaliação vocal assistida EVA foi projetado para o estudo da maioria dos parâmetros de produção da fala, como som, intensidade da voz, pressões aerodinâmicas e por meio de sensores para medir esses parâmetros, o diagnóstico do paciente se torna mais refinado, a fim de realizar um melhor acompanhamento cirúrgico, tratamento medicamentoso ou terapia de voz. A avaliação aerodinâmica é feita por meio da análise das medidas de pressão subglótica, pressão intraoral, fluxo oral, eficiência glótica, eficiência laríngea e resistência laríngea ${ }^{8}$.

Os programas computadorizados para análise acústica de fala e voz se diferem em relação à maneira de calcular os parâmetros acústicos, por isso alguns estudos têm como objetivo padronizar os dados para seus equipamentos ${ }^{9}$. Além disso, esses valores também variam conforme os instrumentos de gravação, ruído ambiental, gênero e idade do falante, o que mostra que a qualidade do equipamento utilizado no registro das vozes, o tipo de programa e as características anatomofuncionais da laringe podem influenciar nos resultados destas medidas em curto prazo ${ }^{10}$.

O objetivo do presente estudo é definir medidas aerodinâmicas em falantes do português brasileiro, sem queixas vocais, obtidas pelo programa EVA.

\section{MÉTODO}

Para realização da pesquisa foram selecionados 40 indivíduos falantes nativos do português brasileiro, sendo 20 do sexo masculino e 20 do sexo feminino. A faixa etária do grupo de estudo foi delimitada entre 18 e 45 anos para eliminar possíveis alterações de voz decorrente do período da muda vocal e da presbifonia. A média de idade dos participantes do sexo feminino foi de 28,65 anos e do sexo masculino 30,25 anos.

Além disso, como critério de inclusão, os participantes foram submetidos à análise perceptivo-auditiva realizada por dois fonoaudiólogos, a fim de certificar que não possuíam alteração na qualidade vocal ou qualquer outro distúrbio da comunicação que impedisse a realização das tarefas propostas.

Todos os participantes foram informados sobre objetivo, procedimentos e divulgação dos resultados do estudo e após a concordância, assinaram o Termo de Consentimento Livre e Esclarecido.

Para a coleta e análise do material, foi utilizado o programa de análise acústica EVA, As gravações foram realizadas no Laboratório de Fonética da UFMG (LABFON) utilizando o computador Dell Vostro 200. Todos os indivíduos tiveram suas vozes gravadas por meio de sinais acústicos e aerodinâmicos, a fim de obter valores de referência para pressão subglótica e fluxo oral. A gravação simultânea de voz e de fluxo aéreo foi possível devido a um equipamento acoplado ao EVA, que consiste em um suporte mecânico do microfone, sensores de fluxo e uma máscara de silicone flexível, o que permite uma vedação necessária para coleta das medidas do fluxo oral. (Figura 1)

Para obtenção da medida da pressão subglótica o indivíduo foi orientado a emitir a frase "Papai papa o papá de Paulo", duas vezes consecutivas. Para análise dos dados considerou-se a emissão da sílaba tônica /pa/ na palavra /papa/, pois o verbo tende a se sobressair prosodicamente em relação ao complemento e, além disso, foi considerado o segundo enunciado, devido a maior estabilidade acústica. 


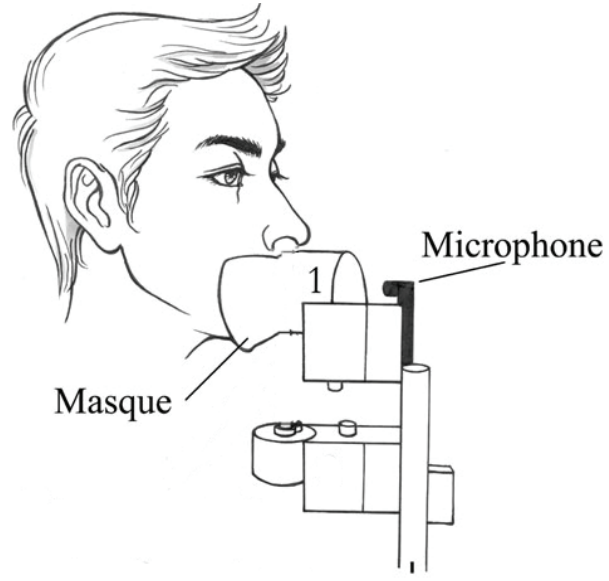

Fonte: http://www.sqlab.fr/

Figura 1 - Dispositivo de EVA
O material de fala utilizado para o estudo foram emissões de palavras e frases que continham características necessárias à análise, a fim de obter as medidas analisadas. O fonema /p/ apresenta características favoráveis à medição da pressão subglótica. Na produção desse fonema os lábios encontram-se fechados e a glote aberta. Há um equilíbrio de pressão estabelecido no trato vocal. A pressão subglótica pode ser estimada pela medição da pressão intra-oral. Durante a produção do fonema /a/, os lábios estão abertos, fator que permite a medida da pressão subglótica. No entanto, o registro da intensidade acústica e o fluxo aéreo oral permitem a obtenção de uma combinação de valores que produzem uma noção da eficiência glótica ${ }^{11}$. (Figura 2)

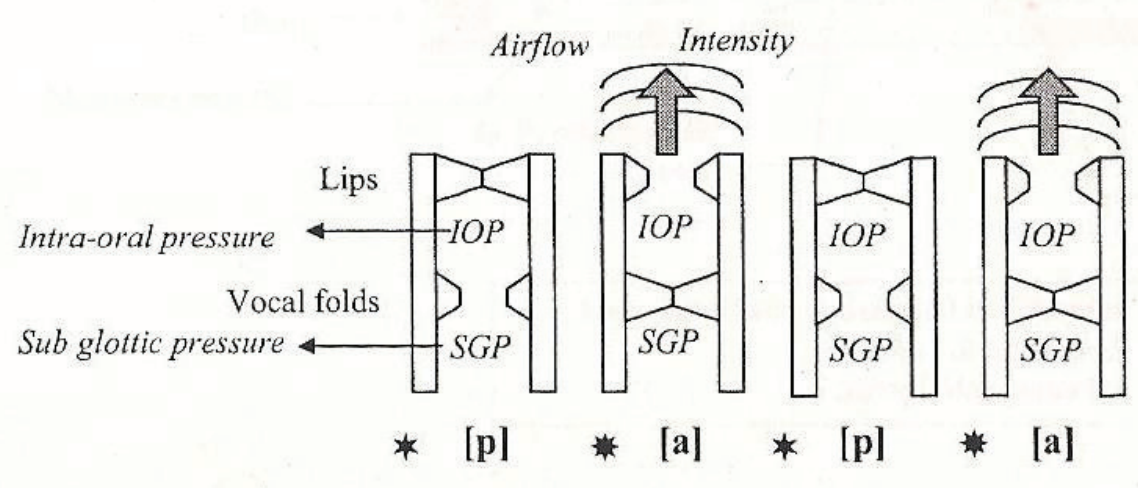

Figura 2 - Representação da pressão intra-oral e pressão subglótica durante a oclusão dos lábios na produção do fonema "p". Sarr et al., (2009) ${ }^{21}$

Para medida do fluxo oral o informante foi instruído a emitir as palavras "patada, pacata, papada, babada, badala e bagaça”, apresentadas em uma folha de papel e lidas pelos informantes. As palavras utilizadas para análise foram a segunda e a terceira, devido à maior estabilidade de emissão.

A medida simultânea do fluxo aéreo oral e da pressão oral determinam a resistência laríngea $(R)$. O fluxo oral é medido por meio de uma máscara facial de silicone colocada sobre a boca do paciente. A máscara é acoplada a um dispositivo que, por sua vez, é conectado a um transdutor de pressão. A pressão intra-oral é medida por meio de um catéter de polietileno de pequeno diâmetro inserido na máscara através de um orifício lateral e posicionado na cavidade oral do paciente. A outra extremidade do catéter é conectada a um transdutor de pressão. A intensidade vocal é captada por meio de um microfone. Os sinais dos transdutores são enviados a um sistema computadorizado. Com base nos dados de pressão oral (pressão subglótica) e do fluxo oral o programa calcula a resistência laríngea, dividindo o valor médio da pressão pelo valor médio do fluxo ${ }^{11}$. (Figura 3 ) 


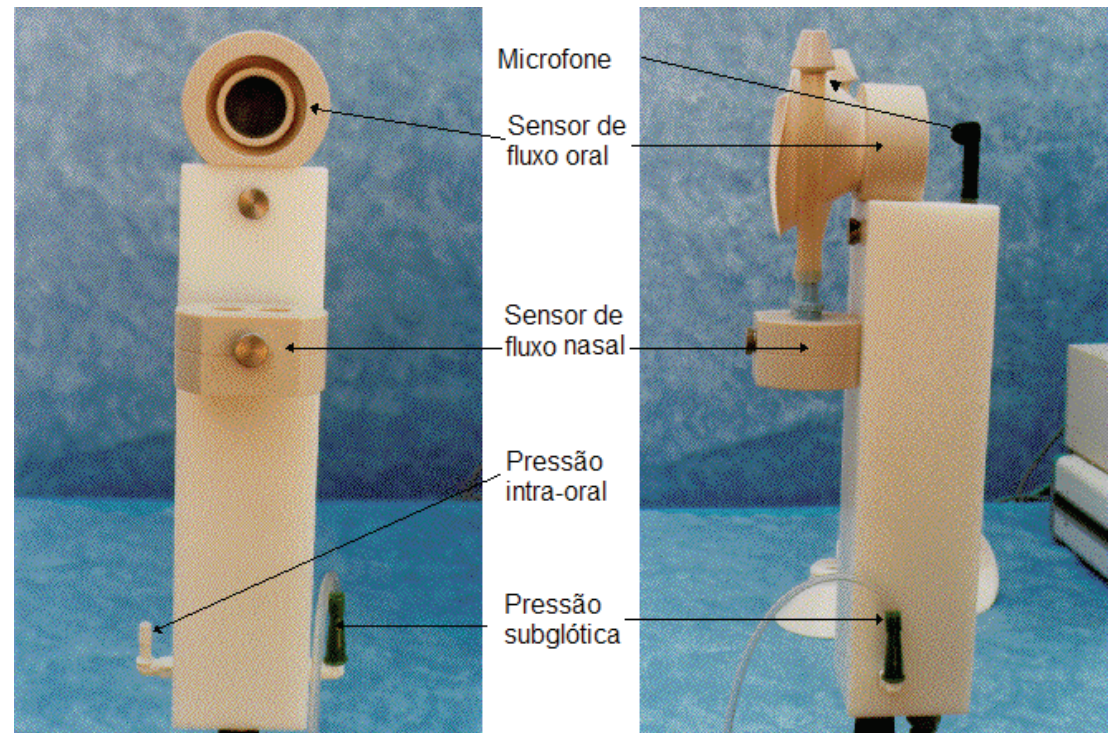

Fonte: http://www.sqlab.fr/

Figura 3 - Equipamento EVA

No material coletado foram analisados os seguintes valores das medidas aerodinâmicas (pressão subglótica e fluxo oral): pressão, média da intensidade, média do fluxo oral, eficiência glótica, eficiência laríngea, resistência laríngea, média da frequência fundamental e fluxo oral do pico de pressão. Foram considerados os valores de cada um destes parâmetros em função do sexo, para obtenção do padrão de normalidade dos falantes nativos do português brasileiro. De acordo com o manual de instrução do programa:

- A Pressão subglótica pode fornecer indicações sobre o esforço necessário para um paciente para iniciar a fonação. A unidade de medida é $\mathrm{hPa}$.

- A intensidade é o resultado da amplitude da onda glótica relacionada à frequência de vibração das pregas vocais. Para a sua realização, três fatores são importantes: pressão de ar subglótica, quantidade de fluxo aéreo e resistência glótica. A unidade de medida é $\mathrm{dB}$.

- O fluxo de ar é frequentemente descrito usando a taxa média de fluxo (MFR), que fornece uma estimativa da impedância da glote e integridade. A unidade de medida é $\mathrm{dm}^{3} / \mathrm{s}$.

- Eficiência glótica é definida pela razão entre intensidade e pressão através da glote. A unidade de medida é $\mathrm{dB} / \mathrm{hPa}$.

- Eficiência laríngea é determinada pela intensidade, dividido pela pressão através da glote e fluxo do ar. A unidade de medida é $\mathrm{dB} / \mathrm{hPa}$. $\mathrm{dm}^{3} / \mathrm{s}$ ).

- Resistência laríngea é um parâmetro semeIhante definido como pressão subglótica dividido pelo fluxo de ar translaríngeo. A unidade de medida é $\mathrm{hPa} /\left(\mathrm{dm}^{3} / \mathrm{s}\right)$.

A média da frequência fundamental fornece uma medida geral do número de ciclos no intervalo de tempo de um segundo. A unidade de medida é Hertz $(\mathrm{Hz})^{6}$.

Este trabalho foi aprovado pelo Comitê de Ética em Pesquisa (COEP) da Universidade Federal de Minas Gerais com o número ETIC 0488.0.203.000-10.

A análise estatística dos dados foi realizada por meio do programa estatístico SPSS (Statistical Package for the Social Sciences) versão 17.0. Primeiramente foi realizada uma análise descritiva dos dados com medidas de tendência central e disperção. Posteriormante, para análise estatística dos valores entre os gêneros foi utilizado o teste não paramétrico para amostras independentes, Mann Whitney. Foi considerado o nível de confiança de $95 \%$.

\section{RESULTADOS}

As tabelas abaixo mostram os valores mínimo, máximo, média, desvio padrão e nível de significância das medidas aerodinâmicas.

A tabela 1 apresenta os valores da pressão subglótica encontrados nos indivíduos do sexo feminino e masculino e nota-se diferença estatisticamente significante referente ao gênero para as medidas de média de intensidade, eficiência laríngea, resistência laríngea e média da frequência fundamental. 
Tabela 1 - Valores das medidas aerodinâmicas de pressão subglótica em indivíduos do sexo feminino e masculino

\begin{tabular}{|c|c|c|c|c|c|c|}
\hline & Gênero & Mínimo & Máximo & DP & Média & $\boldsymbol{P}$ \\
\hline \multirow{2}{*}{$\begin{array}{c}\text { PRESSÃO } \\
\text { SUBGLÓTICA }\end{array}$} & Feminino & 4,00 & 8,20 & 1,09 & 5,84 & \multirow{2}{*}{0,088} \\
\hline & Masculino & 4,60 & 10,90 & 1,62 & 6,70 & \\
\hline \multirow{2}{*}{$\begin{array}{c}\text { MÉDIA DA } \\
\text { INTENSIDADE }\end{array}$} & Feminino & 59,40 & 90,80 & 8,77 & 79,21 & \multirow{2}{*}{0,001} \\
\hline & Masculino & 70,40 & 90,10 & 5,34 & 81,70 & \\
\hline \multirow{2}{*}{$\begin{array}{c}\text { MÉDIA DO FLUXO } \\
\text { ORAL }\end{array}$} & Feminino & 0,03 & 0,19 & 0,04 & 0,09 & \multirow{2}{*}{0,499} \\
\hline & Masculino & 0,07 & 0,57 & 0,11 & 0,16 & \\
\hline \multirow{2}{*}{$\begin{array}{c}\text { EFICIÊNCIA } \\
\text { GLÓTICA }\end{array}$} & Feminino & 10,12 & 16,18 & 1,86 & 13,87 & \multirow{2}{*}{0,152} \\
\hline & Masculino & 8,27 & 18,96 & 3,16 & 12,78 & \\
\hline \multirow{2}{*}{$\begin{array}{l}\text { EFICIÊNCIA } \\
\text { LARÍNGEA }\end{array}$} & Feminino & 68,69 & 420,50 & 91,79 & 188,02 & \multirow{2}{*}{0,000} \\
\hline & Masculino & 26,61 & 164,29 & 40,73 & 97,11 & \\
\hline \multirow{2}{*}{$\begin{array}{l}\text { RESISTÊNCIA } \\
\text { LARÍNGEA }\end{array}$} & Feminino & 36,61 & 330,02 & 71,42 & 96,26 & \multirow{2}{*}{0,005} \\
\hline & Masculino & 10,20 & 132,34 & 27,10 & 54,64 & \\
\hline \multirow{2}{*}{$\begin{array}{c}\text { MÉDIA DA } \\
\text { FREQUENCIA } \\
\text { FUNDAMENTAL }\end{array}$} & Feminino & 178,90 & 257,00 & 20,74 & 208,28 & \multirow{2}{*}{0,000} \\
\hline & Masculino & 102,00 & 279,80 & 38,13 & 136,56 & \\
\hline \multirow{2}{*}{$\begin{array}{c}\text { PICO DA } \\
\text { PRESSÃO DO } \\
\text { FLUXO ORAL }\end{array}$} & Feminino & 0,007 & 1,058 & 0,229 & 0,093 & \multirow{2}{*}{0,741} \\
\hline & Masculino & 0,005 & 0,467 & 0,098 & 0,065 & \\
\hline
\end{tabular}

P-valor <0,05. Teste Estatístico: Teste de Mann-Whitney

Legenda:

DP: desvio padrão

P: nível de significância

A tabela 2 apresenta os valores de fluxo oral dos indivíduos do sexo feminino e masculino. Nota-se diferença estatisticamente significante referente ao gênero apenas para a medida da frequência fundamental, o que já era esperado, visto que se trata de gêneros distintos.

\section{DISCUSSÃO}

A produção da voz está diretamente relacionada com a vibração das pregas vocais, que é gerada pela interação entre o fluxo glotal e o tecido das pregas vocais. Um dos mecanismos importantes na produção vocal é a aerodinâmica, que consiste no estudo do movimento do ar e, de acordo com a mecânica dos fluidos, o ar é constituído de um elevado número de partículas que podem se deslocar livremente umas em relação às outras ${ }^{12}$. Além disso, os fluidos podem se deformar sob a ação de forças arbitrariamente pequenas e quanto menor a força aplicada no fluido, mais lentas serão as deformações ${ }^{13}$.

Existem propriedades do ar que são importantes no estudo da aerodinâmica, sendo elas: volume, pressão, fluxo, viscosidade e massa específica ${ }^{14}$.
O volume é definido como o espaço tridimensional ocupado por uma substância ou corpo ${ }^{13}$. O volume do trato vocal consiste no espaço disponível para o ar, entre o fechamento da glote e a cavidade oral (em consoantes oclusivas), sendo este volume de aproximadamente $3000 \mathrm{~cm}^{3}$ (3 litros) $)^{14}$. O trato vocal é comparado a um tubo que se modifica com a movimentação dos articuladores, em especial, com a movimentação da língua. Estas modificações do trato determinam as regiões de ressonância, ou formantes, correlatos acústicos dos diferentes gestos articulatórios dos sons vocálicos. A mudança do volume está relacionada com a pressão e fluxo de ar no trato vocal ${ }^{15}$.

A pressão, outra propriedade do ar, relevante para o estudo de seu comportamento aerodinâmico, pode ser definida como a força exercida por unidade de área. A pressão intra-oral é medida em relação à pressão atmosférica. Para que haja produção sonora nas pregas vocais é necessário que exista uma diferença mínima de $2 \mathrm{~cm} \mathrm{H}_{2} \mathrm{O}^{3}$ entre as pressões sub e supraglótica ${ }^{15}$.

A lei de Boyle correlaciona pressão e volume, ou seja, de acordo com essa lei, "a pressão de uma dada quantidade de gás cuja temperatura é 
Tabela 2 - Valores das medidas aerodinâmicas do fluxo oral em indivíduos do sexo feminino e masculino

\begin{tabular}{|c|c|c|c|c|c|c|}
\hline & Gênero & Mínimo & Máximo & DP & Média & $P$ \\
\hline \multirow{2}{*}{$\begin{array}{c}\text { PRESSÃO } \\
\text { SUBGLÓTICA }\end{array}$} & Feminino & 3,60 & 8,00 & 1,09 & 6,05 & \multirow{2}{*}{0,675} \\
\hline & Masculino & 1,90 & 10,30 & 2,07 & 6,60 & \\
\hline \multirow{2}{*}{$\begin{array}{c}\text { MÉDIA DA } \\
\text { INTENSIDADE }\end{array}$} & Feminino & 56,40 & 72,60 & 4,75 & 65,50 & \multirow{2}{*}{0,715} \\
\hline & Masculino & 57,10 & 76,60 & 5,62 & 66,30 & \\
\hline \multirow{2}{*}{$\begin{array}{c}\text { MÉDIA DO FLUXO } \\
\text { ORAL }\end{array}$} & Feminino & 0,03 & 0,19 & 0,05 & 0,10 & \multirow{2}{*}{0,106} \\
\hline & Masculino & 0,06 & 0,26 & 0,05 & 0,13 & \\
\hline \multirow{2}{*}{$\begin{array}{c}\text { EFICIÊNCIA } \\
\text { GLÓTICA }\end{array}$} & Feminino & 9,12 & 16,56 & 1,78 & 11,12 & \multirow{2}{*}{0,330} \\
\hline & Masculino & 5,85 & 37,77 & 6,76 & 11,77 & \\
\hline \multirow{2}{*}{$\begin{array}{l}\text { EFICIÊNCIA } \\
\text { LARÍNGEA }\end{array}$} & Feminino & 57,59 & 308,84 & 78,29 & 144,83 & \multirow{2}{*}{0,160} \\
\hline & Masculino & 31,96 & 217,14 & 37,89 & 97,89 & \\
\hline \multirow{2}{*}{$\begin{array}{l}\text { RESISTÊNCIA } \\
\text { LARÍNGEA }\end{array}$} & Feminino & 33,72 & 166,31 & 41,13 & 78,98 & \multirow{2}{*}{0,168} \\
\hline & Masculino & 7,23 & 125,81 & 31,37 & 61,81 & \\
\hline \multirow{2}{*}{$\begin{array}{c}\text { MÉDIA DA } \\
\text { FREQUENCIA } \\
\text { FUNDAMENTAL }\end{array}$} & Feminino & 119,30 & 375,70 & 46,63 & 222,52 & \multirow{2}{*}{0,000} \\
\hline & Masculino & 108,20 & 216,40 & 25,48 & 139,20 & \\
\hline \multirow{2}{*}{$\begin{array}{c}\text { PICO DA } \\
\text { PRESSÃO DO } \\
\text { FLUXO ORAL }\end{array}$} & Feminino & 0,000 & 0,140 & 0,030 & 0,050 & \multirow{2}{*}{0,722} \\
\hline & Masculino & 0,003 & 0,204 & 0,053 & 0,058 & \\
\hline
\end{tabular}

P-valor <0,05. Teste Estatístico: Teste de Mann-Whitney

Legenda:

DP: desvio padrão

P: nível de significância

constante varia inversamente com o volume". O movimento do ar entre duas regiões - por exemplo, a atmosfera e os pulmões - é condicionado pela diferença de pressão entre os dois ${ }^{16}$. Especificamente, o ar fluirá de uma região de pressão alta para uma pressão baixa. Existe uma relação inversamente proporcional entre pressão e volume, ao dobrar a pressão, o volume é reduzido 2 vezes; ao quadruplicar a pressão, o volume é reduzido 4 vezes. Conclui-se que sob temperatura constante, o produto da pressão e do volume de uma massa gasosa é constante ${ }^{13,16}$.

A velocidade é definida como a taxa de variação da distância percorrida por uma partícula no tempo e, a vazão volumétrica é definida como a quantidade (volume) que passa por uma determinada área, em um determinado instante de tempo ${ }^{13}$. Em sons em que o trato vocal encontra-se desobstruído a velocidade do volume de fluxo de ar será menor em relação ao trato obstruído ${ }^{17}$.

De acordo com o Princípio de Bernoulli, a velocidade do fluxo é alta em uma região de estreitamento, ou seja, quando as pregas vocais encontram-se aduzidas, criando queda de pressão dessa região que "aspira" as pregas vocais. Quando o ar é expelido para fora da glote, naquela região se desenvolve uma pressão negativa que aspira as pregas vocais. O quanto isso contribui para o fechamento da glote depende da mobilidade da mucosa da prega vocal. Portanto o aumento da velocidade de escoamento acarreta em queda de pressão ${ }^{18}$.

Com o escape de ar pela glote, a pressão subglótica diminui, diminuindo também a força que mantém as pregas vocais separadas. Com o fechamento da glote, a pressão subglótica volta a aumentar, reiniciando todo o processo. Este ciclo é chamado de ciclo glótico. Ocorre em média 110 vezes por segundo no homem e 200 vezes por segundo na mulher ${ }^{19}$.

Catford, 1977, afirma que existem dois tipos de fluxo: laminar e turbulento. No escoamento laminar as partículas não apresentam mudanças bruscas de direção ou velocidade, e suas linhas de fluxo são paralelas, sem misturas ou flutuações súbitas de velocidade. Já o escoamento turbulento faz com que o fluxo não siga um caminho determinado pelo canal. As camadas de fluido se misturam e há constantes mudanças de direção na velocidade. Não existe um padrão de direção quanto às linhas do fluxo ${ }^{20}$. Os fluxos de ar em altas velocidades, especialmente através de um conduto de paredes irregulares, são geralmente desorganizados, até 
mesmo caóticos, e tendem a formar vórtices e turbilhões que interagem uns aos outros de forma imprevisível. Isto é chamado de fluxo turbulento ${ }^{20}$. Os sons fricativos são produzidos sob um regime de fluxo turbulento, a fricativa ocorre no trato vocal quando um jato veloz de ar atinge um obstáculo. $\mathrm{O}$ ar que sai da constrição ou passa o obstáculo se expande e forma um jato turbulento, produzindo ruído. Quando a velocidade do fluxo de ar aumenta o nível de ruído resultante da turbulência também aumenta ${ }^{17}$.

No presente estudo foi encontrado maior valor de resistência glótica para os indivíduos do sexo feminino quando comparados ao sexo masculino. Esse resultado vai ao encontro com achados em estudo realizado anteriormente. $\mathrm{O}$ autor acredita que, como a resistência é dependente do tamanho da via aérea, o maior valor encontrado para os indivíduos do sexo feminino pode ser atribuído ao fato desses indivíduos apresentarem menor tamanho da laringe quando comparado ao sexo masculino ${ }^{21}$.

Estudos aerodinâmicos realizados com indivíduos normais, ou seja, sem quaisquer alterações funcionais ou anatômicas no trato vocal, obtiveram valores médios de pressão subglótica, fluxo oral e resistência laríngea de $6,8 \pm 1,3 \mathrm{cmH} 2 \mathrm{O}, 145 \pm 44 \mathrm{ml} / \mathrm{s}$ e $49 \pm 14 \mathrm{cmH} 2 \mathrm{O} / \mathrm{l} / \mathrm{s}$, respectivamente ${ }^{22}$. Tais resultados corroboram com o presente estudo (Tabela 1), nos valores médios dos parâmetros de pressão subglótica e resistência laríngea. O fluxo oral apresentou maiores valores quando comparado ao estudo citado.

Um estudo com indivíduos que apresentavam fechamento velofaríngeo marginal obteve como resultado valores médios de resistência laríngea e pressão intra-oral de $39,2 \pm 13,4 \mathrm{cmH} 2 \mathrm{O} / \mathrm{L} / \mathrm{seg}$ e $4,8 \pm 0,8 \mathrm{cmH} 2 \mathrm{O}$, respectivamente ${ }^{23}$. Esses dados corroboram com os resultados encontrados no presente estudo (Tabela 1). É importante salientar que os resultados do presente estudo foram expressos em unidade de medida de pressão em $\mathrm{hPa}$, que $1 \mathrm{hPa}$ equivale à aproximadamente $1 \mathrm{cmH} 2 \mathrm{O} / \mathrm{L} / \mathrm{seg}$

Pesquisas realizadas com falantes franceses apresentou valores médios de pressão subglótica de 6,7 hPa para indivíduos sem alterações vocais, de ambos os sexos. Os resultados apresentados foram próximos em relação ao presente estudo (Tabela 1), mas este obteve médias de pressão subglótica menores em indivíduos do sexo feminino quando comparado ao sexo masculino. Já o fluxo oral foi de $153 \mathrm{~cm}^{3} / \mathrm{s}$ para indivíduos do sexo masculino e $136 \mathrm{~cm}^{3}$ para indivíduos do sexo feminino (Tabela 2). Estes resultados não corroboram com o presente estudo que obteve médias de fluxo oral menor em relação ao estudo referido ${ }^{24}$. Tal fato pode ser justificado considerando que variações linguísticas decorrentes de padrões culturais da língua também afetam os padrões de fala e voz e provocam diferenças significantes de dados acústicos aerodinâmicos ${ }^{9}$.

Estudo aerodinâmicos realizados com falantes franceses, parkinsonianos, todos do sexo masculino apresentou para o grupo controle valores médios de pressão subglótica equivalente a 7,6 $\mathrm{hPa}$, medida no segundo fonema / $/$ / da frase utilizada para coleta dos dados ${ }^{25}$. Esse valor aproxima-se ao apresentado no presente estudo (tabela 1), que obteve média de pressão subglótica equivalente a 6,7 hPa para indivíduos do sexo masculino, obtido no terceiro fonema $/ \mathrm{p} /$ da frase utilizada para obtenção dos dados.

Em relação aos valores que se referem à pressão subglótica, houve diferença estatisticamente significante em relação ao gênero para as medidas de média de intensidade, eficiência laríngea, resistência laríngea e média da frequência fundamental (Tabela 1).

Quanto as medidas de fluxo oral, houve diferença estatisticamente significante em relação ao gênero apenas para a medida da frequência fundamental (Tabela 2).

Não foram encontrados estudos relacionados às medidas aerodinâmicas de eficiência laríngea, eficiência glótica, média da intensidade e da frequência fundamental correlacionando ao gênero. Portanto, não foi possível fazer a comparação dos valores encontrados no presente estudo com achados da literatura.

As diferenças nos programas relacionados à análise acústica fazem com que cada um destes seja único e desta forma impedem a normatização singular. É de grande importância a validação de dados acústicos e padronização de análise, a fim de modificar a subjetividade que tem sido considerada inerente à avaliação da voz humana.

\section{CONCLUSÃO}

Os valores médios referentes à normalidade das medidas aerodinâmicas da pressão subglótica, em falantes do português brasileiro, sem queixas vocais, encontrados no presente estudo para vozes femininas foram: Pressão subglótica= $5,84 \mathrm{hPa}$, média da intensidade $=79,21 \mathrm{~dB}$, média do fluxo oral $=0,09 \mathrm{dm}^{3} / \mathrm{s}$, eficiência glótica $=13,87$ $\mathrm{dB} / \mathrm{hPa}$, eficiência laríngea $=188,08 \mathrm{~dB} /(\mathrm{hPa}$. $\left.\mathrm{dm}^{3} / \mathrm{s}\right)$, resistência laríngea $=96,26 \mathrm{hPa} /\left(\mathrm{dm}^{3} / \mathrm{s}\right)$, média da frequência fundamental $=208,28 \mathrm{~Hz}$ e pico da pressão do fluxo oral=0,093. Os valores encontrados para o sexo masculino foram: Pressão subglótica $=6,7 \mathrm{hPa}$, média da intensidade $=81,7 \mathrm{~dB}$, 
média do fluxo oral $=0,16 \mathrm{dm}^{3} / \mathrm{s}$, eficiência glótica $=12,78 \mathrm{~dB} / \mathrm{hPa}$, eficiência laríngea $=97,11$ $\mathrm{dB} /\left(\mathrm{hPa} \cdot \mathrm{dm}^{3} / \mathrm{s}\right)$, resistência laríngea $=52,64$ $\mathrm{hPa} /\left(\mathrm{dm}^{3} / \mathrm{s}\right)$, média da frequência fundamental= $136,56 \mathrm{~Hz}$ e pico da pressão do fluxo oral $=0,098$.

Os valores médios referentes à normalidade das medidas aerodinâmicas do fluxo oral encontrados no presente estudo, para vozes femininas foram: Pressão subglótica $=6,05 \mathrm{hPa}$, média da intensidade $=65,50 \mathrm{~dB}$, média do fluxo oral $=0,10$ $\mathrm{dm}^{3} / \mathrm{s}$, eficiência glótica $=11,12 \mathrm{~dB} / \mathrm{hPa}$, eficiência laríngea $=144,83 \mathrm{~dB} /\left(\mathrm{hPa} \cdot \mathrm{dm}^{3} / \mathrm{s}\right)$, resistência laríngea $=78,98 \mathrm{hPa} /\left(\mathrm{dm}^{3} / \mathrm{s}\right)$, média da frequência fundamental $=222,52 \mathrm{~Hz}$ e pico da pressão do fluxo oral $=0,047$. Os valores encontrados para o sexo masculino foram: Pressão subglótica $=6,6$
$\mathrm{hPa}$, média da intensidade $=66,3 \mathrm{~dB}$, média do fluxo oral $=0,13 \mathrm{dm}^{3} / \mathrm{s}$, eficiência glótica $=11,77 \mathrm{~dB} /$ $\mathrm{hPa}$, eficiência laríngea $=97,89 \mathrm{~dB} /\left(\mathrm{hPa} \cdot \mathrm{dm}^{3} / \mathrm{s}\right)$, resistência laríngea $=61,81 \mathrm{hPa} /\left(\mathrm{dm}^{3} / \mathrm{s}\right)$, média da frequência fundamental $=139,20 \mathrm{~Hz}$ e pico da pressão do fluxo oral= 0,053.

Em relação aos valores que se referem à pressão subglótica, houve diferença estatisticamente significante em relação ao gênero para as medidas de média de intensidade, eficiência laríngea, resistência laríngea e média da frequência fundamental. Aos valores que se referem ao fluxo oral, houve diferença estatisticamente significante em relação ao gênero apenas para a medida da frequência fundamental.

\begin{abstract}
Purpose: to define aerodynamic measures in Brazilian Portuguese speakers without voice complaints, obtained by the EVA program. Method: the study included 20 men and 20 women who had their voices analyzed. Results: the mean values with subglottic pressure for female and male voices were: Subglottic pressure $=5.84 \mathrm{hPa}$ and $6.7 \mathrm{hPa}$; average intensity $=79.21 \mathrm{~dB}$ and $81.7 \mathrm{~dB}$; oral mean flow $=$ $0.09 \mathrm{dm} 3 / \mathrm{s}$ and $0.16 \mathrm{dm} 3 / \mathrm{s}$; glottic efficiency $=13.87 \mathrm{~dB} / \mathrm{hPa}$ and $12.78 \mathrm{~dB} / \mathrm{hPa}$; laryngeal efficiency = $188.08 \mathrm{~dB} /(\mathrm{hPa} . \mathrm{dm} 3 / \mathrm{s})$ and $97.11 \mathrm{~dB} /(\mathrm{hPa} \cdot \mathrm{dm} 3 / \mathrm{s})$; laryngeal resistance $=96.26 \mathrm{hPa} /(\mathrm{dm} 3 / \mathrm{s})$ and $52.64 \mathrm{hPa} /(\mathrm{dm} 3 / \mathrm{s})$; mean fundamental frequency (F0) $=208.28 \mathrm{~Hz}$ and $136.56 \mathrm{~Hz}$ and peak mouth pressure flow $=0.093$ and 0.098 . There was a statistically significant difference in relation to gender for the measures of average intensity, efficiency, larynx, laryngeal resistance and mean fundamental frequency. The average flow for the oral female and male voices were: subglottic pressure $=6.05 \mathrm{hPa}$ and $6.6 \mathrm{hPa}$; average intensity $=65.50 \mathrm{~dB}$ and $66.3 \mathrm{~dB}$; oral mean flow $=0,10 \mathrm{dm} 3 / \mathrm{s}$ and $0.13 \mathrm{dm} 3 / \mathrm{s}$; glottic efficiency $=11.12 \mathrm{~dB} / \mathrm{hPa}$ and $11.77 \mathrm{~dB} / \mathrm{hPa}$; laryngeal efficiency $=144.83 \mathrm{~dB} /(\mathrm{hPa} . \mathrm{dm} 3 / \mathrm{s})$ and $97.89 \mathrm{~dB} /(\mathrm{hPa} . \mathrm{dm} 3 / \mathrm{s})$; laryngeal resistance $=78.98 \mathrm{hPa} /(\mathrm{dm} 3 / \mathrm{s})$ and $61.81 \mathrm{hPa} /(\mathrm{dm} 3 / \mathrm{s})$; average $\mathrm{FO}=222.52 \mathrm{~Hz}$ and $139.20 \mathrm{~Hz}$ and peak pressure oral flow $=0.047$ and 0.053 . Conclusion: EVA is a new program in Brazil, and analysing the aerodynamic measures in Brazilian Portuguese speakers, allows us to obtain reference values, thus allowing comparisons with future studies.
\end{abstract}

KEYWORDS: Voice; Air Pressure; Speech Acoustics, Speech, Language and Hearing Sciences

\section{REFERÊNCIA}

1. Cappellari VM, Cielo CA. Características vocais acústicas de crianças pré-escolares. Rev Bras Otorrinolaringol. 2008; 74(2): 265-72

2. Gama ACC, Behlau, MS. Estudo da constância de medidas acústicas de vogais prolongadas e consecutivas em mulheres sem queixa de voz e em mulheres com disfonia. Rev Soc Bras Fonoaudiol. 2009; 14(1):8-14.

3. Baggott CD, Yuen AK, Hoffman MR, Zhou L, Jiang JJ. Estimating subglottal pressure via airflow redirection. Laryngoscope. 2007; 117:1491-5.
4. Teston, B. L'étude instrumentale des gestes dans la production de la parole: Importance de l'aérophonométrie. In: P. Auzou; V. RollandMonnoury, S. Pinto, C. Ozsancak (ed.) Les Dysarthries. Marseille: Solal. 2007, p. 115-7.

5. Teles VC, Rosinha ACU. Análise Acústica dos Formantes e das Medidas de Perturbação do Sinal Sonoro em Mulheres Sem Queixas Vocais, Não Fumantes e Não Etilista. Arq. Int. Otorrinolaringol. 2008;12(4):523-30.

6. Rieves AL, Hoffman MR, Jiang JJ. Indirect estimation of laryngeal resistance via airflow redirection. Ann Otol Rhinol Laryngol. 2009; 118(2): 124-30. 
7. Lanziani FF, Yamashita RP, Fukushiro AP, Trindade IEK. Correlação entre fechamento velofaríngeo e dimensões nasofaríngeas após cirurgia de retalho faríngeo avaliados por meio da técnica fluxo-pressão. Rev Soc Bras Fonoaudiol. 2010;15(2):250-5.

8. Ghio A. SQLab. Disponível em: <http://www. sqlab.fr/> Acesso em: 11 de junho de 2011.

9. Felippe ACN, Grillo MHMM, Grechi TH. Normatização de medidas acústicas para vozes normais. Rev Bras Otorrinolaringol. 2006; 72(5): 659-64.

10. Valentim AF, Côrtes MG, Gama, ACC. Análise espectrográfica da voz: efeito do treinamento visual na confiabilidade da avaliação. Rev Soc Bras Fonoaudiol. 2010;15(3):335-42.

11. Brustello CMB, Fukushiro AP, Yamashita RP. Resistência laríngea em indivíduos com fechamento velofaríngeo marginal. Rev Soc Bras Fonoaudiol. 2010;15(1):63-71.

12. Kucinschi BR, Afjeh AA, Scherer RC. On the application of the lattice Boltzmann method to the investigation of glottal flow. J. Acoust Soc Am. 2008; 124(1):523-34.

13. Fox RW, Mcdonald AT, Printchard PJ. Introdução à mecânica dos fluidos. 6. ed. Rio de Janeiro: LTC; 2006. p.5-30

14. Catford JC. Fundamental problems in phonetics. Edinburgh: Edinburgh University Press, 1977. p. 1-13

15. Shosted RK. The Aeroacoustics of Nasalized Fricatives [tese]. Berkeley: University of Califórnia; 2006.

16. Douglas CR. Fisiologia aplicada à Fonoaudiologia. $2^{\underline{a}}$ ed. São Paulo: Guanabara Koogan; 2007.p. $672-95$
17. Shosted RK. Investigating the aerodynamics of nasalized fricatives. Saarbrücken. 2007; 255-60.

18. Zimmer V, Cielo CA, Finger LS. Modificações vocais acústicas espectrográficas produzidas pela fonação reversa. Rev. CEFAC. 2010; 12(4): 535-42. 19. Ferreira LP, Befi-Lopes DM. Tratado de Fonoaudiologia. $1^{\underline{a}}$ ed. São Paulo: Roca; 2004. p.693-700

20. Howe MS, Mcgowan RS. Analysis of flowstructure coupling in a mechanical model of the vocal folds and the subglottal system. J.Fluids Structures. 2009; 25(8): 1299-317.

21. Netsell R, Lotz WK, Duchane AS, Barlow SM. Vocal tract aerodynamics during syllable productions: Normative data and theoretical implications. J Voice. 1991; 5(1):1-9.

22. Yamashita RD, Carlais LL, Miguel HC, Trindade IE. Avaliação da resistência laríngea em indivíduos portadores de insuficiência velofaríngea com distúrbio articulatório compensatório. Acta ORL. 2006; 24(4): 263-7

23. Zlotnicki, V, Wahr J, Fukumori I, Yuhe T. Antarctic Circumpolar Current Transport Variability during 2003-05 from GRACE. J. Phys. Oceanogr.2007; 37(2): 230-44.

24. Ghio A, Pouchoulin G, Giovanni A, Fredouille C, Teston B, Révis J, et. al. "Marchal, a. Approches complémentaires pour l'évaluation des dysphonies: bilan méthodologique et perspectives". Travaux Interdisciplinaires du Laboratoire Parole et Langage d'Aix-en-Provence, vol. 26. 2008, p. 33-74.

25. Sarr MM, Pinto S, Jankowski L, Teston B, Purson A, Ghio A, et. al. Contribution de la mesure de la pression intra-orale pour La compre'hension des troubles de la coordination pneumophonique dans la dysarthrie parkinsonienne. Revue Neurologique. 2009; 165: 1055-61.
http://dx.doi.org/10.1590/S1516-18462012005000053

RECEBIDO EM: 29/09/2011

ACEITO EM: 24/11/2011

Endereço para correspondência:

Karina Vitor de Oliveira

Av. Prof. Alfredo Balena, 190

Belo Horizonte - MG - Brasil

CEP: 30130-100

E-mail: karinavitor19@gmail.com.br 